\title{
Correlation Method of Search and Measurement of Coordinates the Radiatingradio-Electronic Means in the Set Sector
}

\author{
Yuriy L. Koziratsky, \\ Maxim L. Parinov and Ervant A. Mamadjanyan* \\ Military Education and Research Centre of Military-Air Forces \\ «Military-Air Academy \\ Named After Professor N.E. Zhukovsky and Yu.A. Gagarin» \\ 54a Starykh Bolshevikov Str., Voronezh, 394064, Russia
}

Received 20.06.2016, received in revised form 15.08.2016, accepted 24.09.2016

The method correlation Measurement of coordinates the radio emission sources, based on a triangulable way of the fixing is offered, allowing to realize depression of an entrance stream of electromagnetic contacts with RIS to a spatial sign.

Keywords: a radio emission source, search, Measurement of coordinates, a triangulable method of a fixing, correlation processing, narrow-band signals.

Citation: Koziratsky Yu.L., Parinov M.L., Mamadjanyan E.A. Correlation method of search and measurement of coordinates the radiatingradio-electronic means in the set sector, J. Sib. Fed. Univ. Eng. technol., 2016, 9(8), 1247-1253. DOI: $10.17516 / 1999-494 X-2016-9-8-1247-1253$

(c) Siberian Federal University. All rights reserved

* Corresponding author E-mail address: pml_27_2_8@mail.ru 


\title{
Корреляционный метод поиска
}

\section{и координатометрии излучающего \\ радиоэлектронного средства}

\section{в заданном секторе}

\author{
Ю.Л. Козирацкий, \\ М.Л. Паринов, Е.А. Мамаджанян \\ Военный учебно-научный центр Военно-воздушных сил \\ «Военно-воздушная академия \\ имени профессора Н.Е. Жуковского и Ю.А. Гагарина» \\ Россия, 394064, Воронеж, ул. Старых Большевиков, 54 а
}

Предложен метод корреляциионной координатометрии источников радиоизлучения, основанный на триангуляционном способе местоопределения, позволяющий реализовать разрежение входного потока электромагнитных контактов с источником радиоизлучения (ИРИ) по пространственному признаку.

Ключевые слова: источник радиоизлучения, поиск, координатометрия, триангуляционный метод местоопределения, корреляционная обработка, узкополосные сигналы.

Динамические параметры реализации разведывательных процедур, в частности, касающиеся определения местоположения источников излучения, напрямую зависят от плотности потока контактов с излучающими радиоэлектронными средствами и пропускной способности пеленгаторных постов системы местоопределения [1]. Наиболее актуален данный фактор применительно к коротковолновой и ультракоротковолновой части радиочастотного спектра, характеризуемой высокой степенью загруженности диапазона.

Снижение динамических показателей разведывательных средств напрямую влияет на боевую эффективность основных систем вооружения и, как следствие, на исход вооруженного противостояния в целом.

Одним из путей снижения временных затрат на обработку и анализ входного потока контактов с излучающими РЭС является реализация процедур разрежения по пространственному признаку.

Исходя из сказанного выше, цель статьи - формирование предложений и предварительный анализ возможности реализации метода триангуляционного определения местоположения источников радиоизлучений, реализующего разрежение входного потока излучений по пространственному признаку за счет корреляционного анализа наблюдаемых реализаций сигнальных функций ИРИ.

Геометрия задачи местоопределения, основанного на реализации угломерного способа координатометрии при ограничениях на область пространства поиска, представлена на рис. 1, где $D_{x} \times D_{y}$ - геометрические размеры области поиска; $x_{i}, y_{i}$ - координаты геометрического «центра» элемента сектора (области) поиска; $S_{x_{i}, y_{i}}$-элементарный участок области поиска, размеры которого определяются степенью пространственного разрешения пеленгаторной пары, зависящей от вида и ширины диаграмм направленностей антенн пеленгаторов, а также удаленности

$$
-1248-
$$




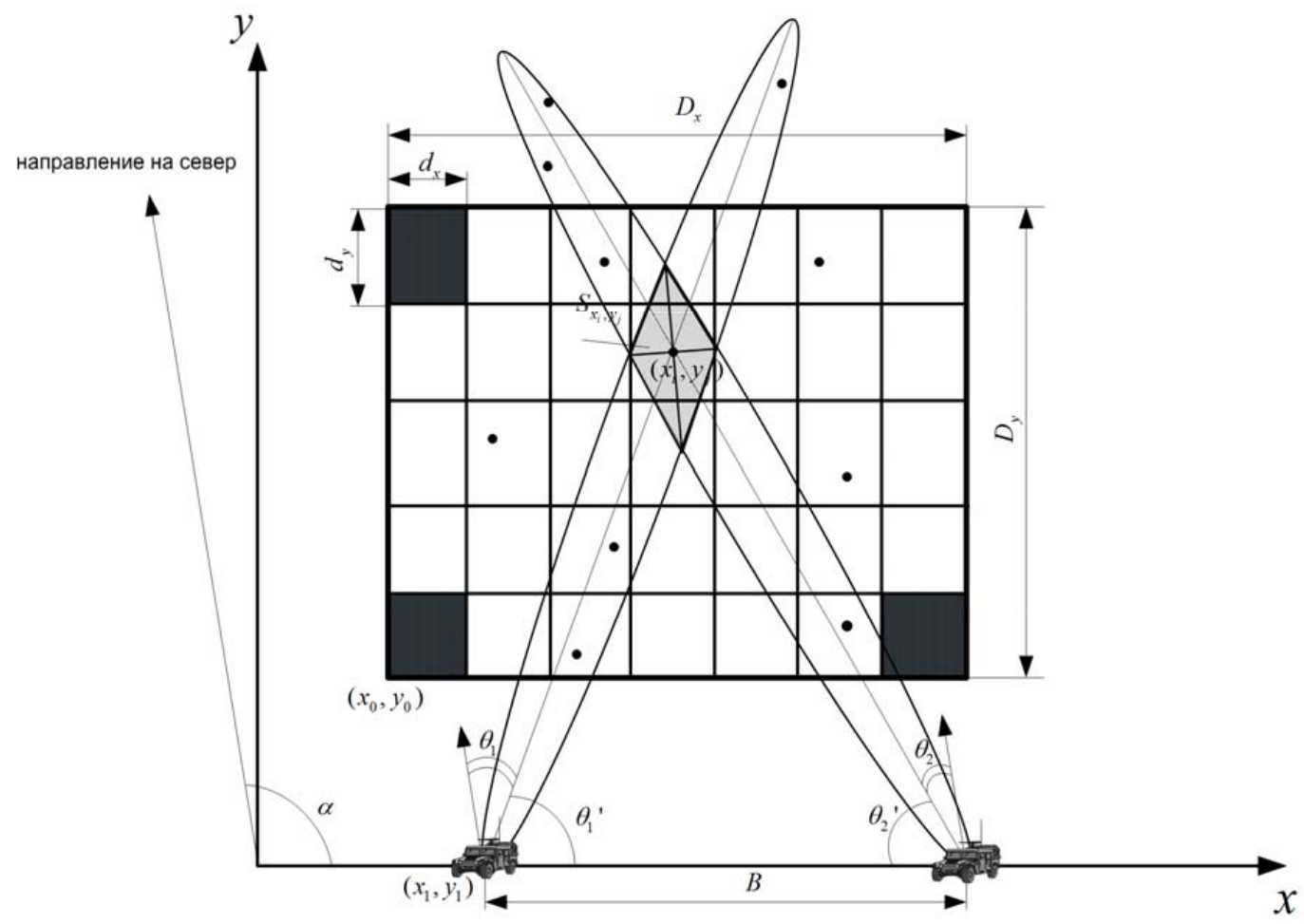

Рис. 1. Графическая постановка задачи

от пеленгаторов; $\theta_{1}, \theta_{2}$ - азимуты направления максимумов диаграмм направленностей антенн первого и второго пеленгаторов соответственно.

Существо триангуляционно-корреляционного местоопределения заключается в реализации синхронного по пространству и времени пеленгования источников излучения с последующей корреляционной обработкой потока сигналов от каждого из пеленгаторов в интересах выявления сигналов тех источников, координаты которых принадлежат априорно заданной «просматриваемой» области пространства [2-4]. Пространственно-временная синхронизация реализуется путем одновременного формирования диаграмм направленности пеленгаторов, максимумы которых ориентированы на геометрический центр просматриваемого элемента области поиска.

Выражения, определяющие вычислительный алгоритм управления диаграммами направленностей пеленгаторов, представлены в следующем виде:

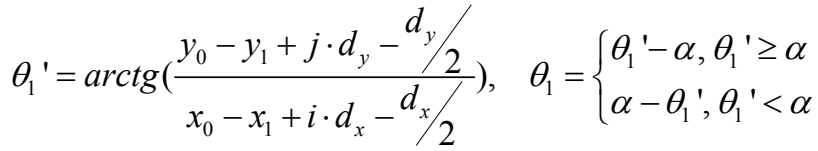

$$
\begin{aligned}
& \theta_{2}{ }^{\prime}=\operatorname{arctg}\left(\frac{y_{0}-y_{1}+j \cdot d_{y}-d_{y} / 2}{x_{1}-x_{0}+B-i \cdot d_{x}+d_{x} / 2}\right), \quad \theta_{2}=\left\{\begin{array}{l}
\theta_{2}{ }^{\prime}+\alpha-\pi, \theta_{2}{ }^{\prime} \geq \pi-\alpha \\
\pi-\left(\alpha+\theta_{2}{ }^{\prime}\right), \theta_{2}{ }^{\prime}<\pi-\alpha
\end{array},\right.
\end{aligned}
$$




$$
\begin{aligned}
& i=\overline{1, n-1}, j=\overline{1, m-1}, \\
& n=\left[\frac{D_{x}}{d_{x}}\right], m=\left[\frac{D_{y}}{d_{y}}\right]
\end{aligned}
$$

Следует отметить, что углы $\mathrm{q}_{1}{ }^{\prime}, \mathrm{q}_{2}{ }^{\prime}$ - величины, функционально зависящие от времени. Вид зависимости определяется выражениями (1) через параметры индексов элементов разрешения области поиска. В частности,

$$
\begin{aligned}
& j=F_{y}(t), \quad E\left\{F_{y}(t)\right\}=[1, m-1] . \\
& i=F_{x}(t), \quad E\left\{F_{x}(t)\right\}=[1, n-1]
\end{aligned}
$$

Одной из основных особенностей формирования $F_{y}(t)$ и $F_{x}(t)$ помимо согласования их областей определения, что обеспечивает синхронизацию в изменении диаграмм направленностей (ДН) пеленгаторов, является реализация возможности функционального исключения элементов разрешения области поиска с наименее вероятным расположением излучающих РЭС.

Очевидно, что с каждого из направлений ориентирования ДН пеленгаторов будет формироваться поток сигналов, излучающих РЭС. Определение наличия в каждом из потоков сигнала одного и того же радиоэлектронного средства, что свидетельствует о его расположении в области пространства пересечения диаграмм направленностей, возможно за счет корреляционной обработки.

При работе источников излучения в непрерывном режиме значение корреляционной функции сигналов пеленгаторов будет определяться в следующем виде:

$$
\begin{aligned}
& K\left(t^{\prime}, t, \mathrm{t}\right)=\int_{t}^{t+\mathrm{t}} \mathrm{x}_{1}(t) \mathrm{x}_{2}\left(t-t^{\prime}\right) d t \\
& \mathrm{x}_{1}(t)=\mathrm{q} S_{1}^{x_{i}, y_{j}}(t)+\sum_{m} S_{m}(t)+n_{1}(t) \\
& \mathrm{x}_{2}(t)=\mathrm{q} S_{2}^{x_{i}, y_{j}}(t)+\sum_{k} S_{k}(t)+n_{2}(t),
\end{aligned}
$$

где q $\in[0,1]$ - случайный параметр различения; $S_{1(2)}^{x_{i}, y_{j}}(t)$ - реализация сигнала $S_{1}(t)$ (искомого сигнала) на выходе первого и второго пеленгаторов соответственно; $S_{m(t)}$ - сигналы, поступающие с фиксированных направлений пеленгования первого и второго пеленгаторов соответственно; $n_{1(2)}(t)$ - шум на выходе первого и второго пеленгаторов; $m=\overline{1, M} ; n=\overline{1, N}$ - количество сигналов в каждом из потоков; $\tau$ - интервал времени накопления.

В интересах решения задачи различения сигналов пороговый уровень принятия решения о наличии в реализациях сигнальных функций сигнала $S^{x_{i}, y_{j}}(t)$ целесообразно определить в соответствии с критерием идеального наблюдателя, предусматривающим минимизацию вероятности суммарной ошибки:

$$
h_{\text {opt }}=\arg \min \left\{P_{\text {Eou }}(h)\right\},
$$

где $P_{\text {гоu }}(h)=\int_{h}^{\infty} p\left(K\left(t^{\prime}, t, \tau\right) / \theta=0\right) d K\left(t^{\prime}, t, \tau\right)+\int_{-\infty}^{h} p\left(K\left(t^{\prime}, t, \tau\right) / \theta=1\right) d K\left(t^{\prime}, t, \tau\right)[5]$ 
Рассмотрим случай пеленгования РЭС, излучающих узкополосные сигналы, поступающие на вход каждого из пеленгаторов. В качестве математических моделей определим комплексные сигналы в виде

$$
\begin{aligned}
& \dot{S}_{1}(t)=S_{m 1}(t) e^{j\left(\omega_{0} t+\varphi_{1}(t)+\varphi_{01}\right)}=\dot{S}_{m 1}(t) e^{j \omega_{0} t} \\
& \dot{S}_{2}(t)=S_{m 2}(t) e^{j\left(\omega_{0} t+\varphi_{2}(t)+\varphi_{02}\right)}=\dot{S}_{m 2}(t) e^{j \omega_{0} t}
\end{aligned}
$$

где $\dot{S}_{m 1}(t), \dot{S}_{m 2}(t)$ - комплексные амплитуды рассматриваемых сигналов пеленгаторов [6].

Исходя из соотношения $a(t) \cdot b(t)=\frac{1}{2} \operatorname{Re}\{\dot{a}(t) \cdot \dot{b}(t)+\dot{a}(t) \cdot \stackrel{*}{b}(t)\}$, можно показать, что

$$
K\left(t^{\prime}, t, \mathrm{t}\right)=\frac{1}{2}\left[\operatorname{Re}\left\{\int_{t}^{t+\mathrm{t}} \dot{S}_{1}(t) \dot{S}_{2}\left(t-t^{\prime}\right) d t\right\}+\operatorname{Re}\left\{\int_{t}^{t+\mathrm{t}} \dot{S}_{1}(t) \stackrel{*}{S}_{2}\left(t-t^{\prime}\right) d t\right\}\right]
$$

Интегрируя комплексные функции сигналов, получим:

$$
\begin{aligned}
& \int_{t}^{t+\mathrm{t}} \dot{S}_{1}(t) \dot{S}_{2}\left(t-t^{\prime}\right) d t=\frac{\dot{S}_{1 m}(t) \dot{S}_{2 m}(t)}{2 \mathrm{w}_{0}}\left[e^{j\left(2 \mathrm{w}_{0} t+\mathrm{w}_{0} t^{t}\right)}\left(e^{j \mathrm{w}_{0} \mathrm{t}}-1\right)\right] \\
& \int_{t}^{t+\mathrm{t}} \dot{S}_{1}(t) S_{2}\left(t-t^{\prime}\right) d t=\dot{S}_{1 m}(t) \stackrel{*}{S}_{2 m}(t) \mathrm{t} e^{-j\left(\mathrm{w}_{0} t_{1}-\Delta \mathrm{j}\right)}
\end{aligned} .
$$

Следует отметить, что выражения (8) получены при ограничениях на динамику изменения огибающей сигналов и множителей угловой модуляции $\left(e^{j_{1}(t)}, e^{j j_{2}(t)}\right)$, а именно

$$
\begin{aligned}
& \left.\frac{d S_{1 m}(t)}{d t}\right|_{t \in[t, t+\mathrm{t}]} \approx 0,\left.\frac{d S_{2 m}(t)}{d t}\right|_{t \in[t, t+\mathrm{t}]} \approx 0 \\
& \left.\frac{d \mathrm{j}_{1}(t)}{d t}\right|_{t \in[t, t+\mathrm{t}]} \approx 0,\left.\frac{d \mathbf{j}_{2}(t)}{d t}\right|_{t \in[t, t+\mathrm{t}]} \approx 0
\end{aligned}
$$

Подставляя (8) в (7), окончательно для корреляционной функции узкополосных сигналов получим:

$$
\begin{gathered}
K\left(t^{\prime}, t, \mathrm{t}\right)=\frac{S_{1 m} S_{2 m}}{2}\left[\frac{\sin \left(\mathrm{w}_{0} \mathrm{t}\right)}{\mathrm{w}_{0}}\right. \\
\sin \left(2 \mathrm{w}_{0} t+\mathrm{w}_{0} \mathrm{t}+\mathrm{w}_{0} t^{\prime}+\mathrm{j}_{1}(t)+\mathrm{j}_{2}(t)+\mathrm{j}_{01}+\mathrm{j}_{02}\right)+ \\
\left.+\mathrm{t} \cos \left(\mathrm{w}_{0} t^{\prime}-\Delta \mathrm{j}\right)\right]
\end{gathered}
$$

Введенные ограничения (9) позволяют произвести анализ корреляционной функции, положив $\mathrm{j}_{1}(t)=\mathrm{j}_{1}=0, \mathrm{j}_{2}(t)=\mathbf{j}_{2}=0, \mathbf{j}_{01}=0, \mathbf{j}_{02}=0$, что не повлияет на закономерность ее поведения на множестве аргументов.

Таким образом,

$$
K\left(t^{\prime}, t, \mathrm{t}\right)=\frac{S_{1 m} S_{2 m}}{2}\left[\frac{\sin \left(\mathrm{w}_{0} \mathrm{t}\right)}{\mathrm{w}_{0}} \sin \left(2 \mathrm{w}_{0} t+\mathrm{w}_{0} \mathrm{t}+\mathrm{w}_{0} t^{\prime}\right)+\mathrm{t} \cos \left(\mathrm{w}_{0} t^{\prime}\right)\right] .
$$

В соответствии с выражением (11) были получены графики зависимости $K\left(t^{\prime}, t, \mathrm{t}\right)$ от времени задержки и интервала времени накопления применительно к сигналу с частотой $f=1 M Г u$ (рис. 2).

Анализ представленных зависимостей свидетельствует о периодичности корреляционной функции, что объясняется гармоническим характером исходных сигналов. При этом в области 

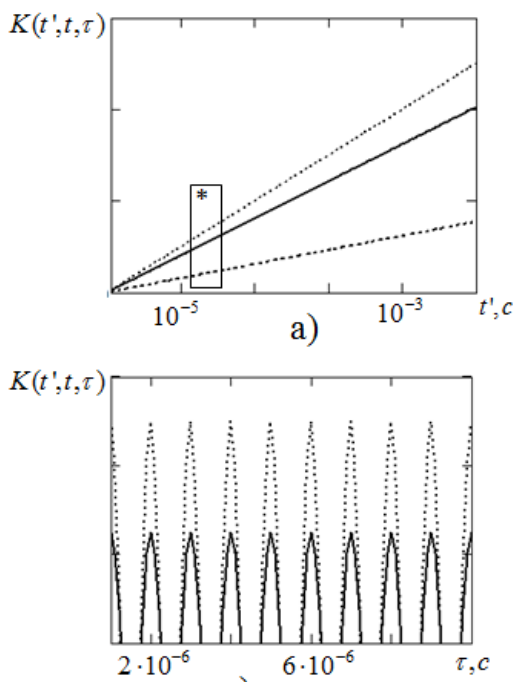

в)

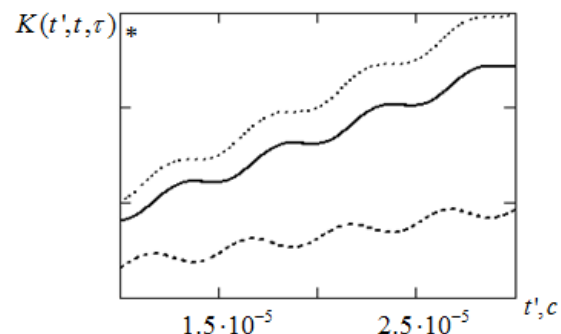

б)

Рис. 2. Графики зависимости $K\left(t^{\prime}, t, \mathrm{t}\right)$ от времени задержки сигналов и времени накопления

значений времени накопления период функции корреляции $T_{K\left(t^{\prime}, t, \mathrm{t}\right)}=T_{0} / 2$ (рис. 2б), в области значений времени задержки между сигналами $T_{K\left(t^{\prime}, t, \mathrm{t}\right)}=T_{0}$ (рис. 26 ). Следует отметить, что при больших значениях времени накопления по сравнению с длительностью периода функции $\left(\mathrm{t}>>T_{0}\right.$ ) величина флуктуаций корреляционной функции значительно меньше ее среднего, так, при $\mathrm{t} / T_{0}=100$ и времени задержки, кратному $T_{0}\left(t_{1} / T_{0}=n, n \in \square\right)-\frac{\overline{K\left(t^{\prime}, t, \mathrm{t}\right)}}{\overline{\Delta K\left(t^{\prime}, t, \mathrm{t}\right)}} \square 1000$ . Помимо этого, периодичность функции корреляции следует учитывать при анализе времени задержки сигналов пеленгаторов, в частности при $t_{1} / T_{0}=\frac{(2 n+1)}{4}, n \in \square-K\left(t^{\prime}, t, \mathrm{t}\right)=0$.

Таким образом, в статье рассмотрен метод определения местоположения источников радиоизлучения в заданном секторе поиска, основанный на триангуляционной координатометрии с совместной корреляционной обработкой аддитивного потока сигнальных функций каждого из пеленгаторных постов, позволяющей реализовать режекцию входного потока электромагнитных контактов с ИРИ, тем самым повысить пропускную способность системы местоопределения и обеспечить поиск и анализ излучений в априорно заданном секторе поиска.

\section{Список литературы}

[1] Радзиевский В.Г., Сирота А.А. Информащионное обеспечение радиоэлектронных систем. М.: ИПРЖР, 2001. 456 c. [Radzievskii V.G., Sirota A.A. Information support of radio-electronic systems. Moscow, IPRZhR, 2001. 456 p. (in Russian)]

[2] Козирацкий Ю.Л., Паринов М.Л. Способ определения местоположения источников радиоизлучений с режекцией входного потока излучений на основе корреляционного анализа 
результатов наблюдения. Радиотехника, 2013, 7, 16-19 [Koziratskii Iu.L., Parinov M.L. A method of determining location of radio emission sources with riccia input stream of radiation based on the correlation analysis of the results of monitoring. Radiotekhnika, 2013, 7, 16-19 (in Russian)]

[3] Патент на изобретение РФ № 2540126, МКИ G01S. М.: РОСПАТЕНТ, 2014 [Patent RF №2540126, MKI G01S. Moscow, Rospatent, 2014 (in Russian)]

[4] Модели пространственного и частотного поиска / отв. ред. Ю.Л. Козирацкий. М.: Радиотехника, 2014. 342 с. [Model of spatial and frequency search. Ed. by Iu.L.Koziratskii. Moscow, Radiotekhnika, 2014. 342 p. (in Russian)]

[5] Тихонов В.И. Статистическая радиотехника. М.: Сов. радио, 1966. 624 с. [Tikhonov V.I. Statistical radio engineering. Moscow, Sov. radio, 1966. 624 p. (in Russian)]

[6] Баскаков С.И. Радиотехнические цепи и сигналы. М.: Высш. шк., 2003. 462 с. [Baskakov S.I. Radio circuits and signals. Moscow, Vysshaia shkola, 2003. 462] 